\title{
ABONO ORGÁNICO, MICROORGANISMOS DE MONTAÑA (MM) Y FERTIBIOL PARA EL CONTROL BIOLÓGICO DE LA HERNIA DE LAS CRUCÍFERAS (Plasmodiophora brassicae wor.) EN EL CULTIVO DE MOSTAZA CHINA (Brassica rapa sp. pekinensis var. Taranko F1)*
}

\author{
Leida Castro-Barquero ${ }^{1 / * *}$, Verónica Martínez-Vargas ${ }^{2}$, \\ Oscar Castro-Zuñiga ${ }^{3}$, Mónica Blanco-Meneses ${ }^{4}$
}

\begin{abstract}
Palabras clave: Plasmodiophora brassicae; hernia de las crucíferas; microorganismos de montaña (MM); abono orgánico; fertibiol; control biológico.

Keywords: Plasmodiophora brassicae; cabbage clubroot; mountain microorganisms (MM); organic matter; fertibiol; biological control.
\end{abstract}

Recibido: 28/11/2019
Aceptado: 04/02/2020

\section{RESUMEN}

Introducción. La producción de mostaza (Brassica rapa sp. pekinensis) se ve afectada por la enfermedad causada por Plasmodiophora brassicae. Objetivo. Determinar el efecto de Abono Orgánico, Microorganismos de Montaña y Fertibiol, para el control biológico de P. brassicae. Materiales y métodos. Los tratamientos diseñados se evaluaron de manera individual y en combinación, las variables evaluadas fueron peso fresco y seco de la parte aérea y la radical, relación raíz-tallo, índice de severidad radical y persistencia de esporas en el suelo (rtPCR). Se realizaron 2 ciclos de cultivo y 2 evaluaciones. Se utilizaron las pruebas de Kruscal-Wallis, Shapiro-Wilks y Levene, ANDEVA y la prueba DGC (Di Rienzo, Guzmán y Casanoves) para la separación de medias. Resultados. Los tratamientos que contenían abono orgánico (solo o
Use of Organic Matter, Mountain Microorganisms (MM) and Fertibiol for the biological control of cabbage club root (Plasmodiophora brassicae wor.) in the cultivation of chinese mustard (Brassica rapa sp. Pekinensis var. Taranko F1). Introduction. Mustard production (Brassica rapa sp. Pekinensis) is affected by the disease caused by Plasmodiophora brassicae. Objective. To determine the effect of Organic Matter, Mountain Microorganisms and Fertibiol, for the biological control of $P$. brassicae. Materials and methods. The designed treatments were evaluated individually and in combination, the variables evaluated were fresh and dry aerial and radical weight, root-stem ratio $(\mathrm{R} / \mathrm{T})$, radical severity index and spore persistence in the soil (rtPCR). Two culture cycles and 2 evaluations

\footnotetext{
* Este trabajo formó parte de la tesis de licenciatura de la segunda autora bajo el marco del proyecto VI-813-B5-092.

** Autora para correspondencia. Correo electrónico: leida.castro@ucr.ac.cr

1 Universidad de Costa Rica, Centro de Investigaciones Agronómicas (CIA), Costa Rica. (D) 0000-0002-3788-7173.
} 
en mezcla) obtuvieron los mayores valores para las variables de peso fresco y seco, tanto para la parte aérea (más de $300 \%$ respecto al testigo) como la radical. La relación raíz-tallo $(\mathrm{R} / \mathrm{T})$ fue menor y, por ende, más favorable en el tratamiento con abono orgánico, con un valor de 0,22 . Para el índice de severidad, el tratamiento con MM presentó el menor valor en las 2 evaluaciones, mientras que los tratamientos con abono orgánico mostraron el mayor valor, pero esto no afectó negativamente el rendimiento. Para la variable persistencia, fue posible determinar que en el testigo hubo una disminución en la cantidad de esporas germinativas con el avance del tiempo y que los tratamientos aplicados ocasionaron respuestas variables en cuanto a este comportamiento, mostrando ecuaciones con diferente pendiente y muy diversos coeficientes de variación. El tratamiento con MM presentó para la segunda evaluación la menor cantidad promedio de esporas y el menor índice de severidad radical. Conclusión. El abono orgánico mejoró el rendimiento de las plantas y la utilización de MM redujo la severidad y mostró potencial para disminuir la presencia de esporas en el suelo.

\section{INTRODUCCIÓN}

La mostaza (Brassica rapa sp. pekinensis) es una hortaliza originaria de China que pertenece a la familia Brassicaceae. En Costa Rica, su cultivo se realiza en su mayoría en las zonas altas de Cartago y Zarcero, entre los 700 y los 2000 msnm, posee un alto consumo en la población costarricense, lo que determina su importancia (Ramírez y Nienhuis 2012).

Una de las enfermedades que limita la producción comercial de brásicas en general were performed. The Kruscal-Wallis, ShapiroWilks and Levene, ANDEVA tests and the DGC (Di Rienzo, Guzmán \& Casanoves) test for the separation of means were used. Results. The treatments containing organic matter obtained the highest values for the variables of fresh weight and dry weight, both for the aerial part (more than 300\% compared to the control) and the radical part. The $\mathrm{R} / \mathrm{T}$ ratio was lower, and therefore more favorable, in the treatment with organic matter, with a value of 0.22 . For the severity index, the MM treatment had the lowest value in the 2 evaluations, while the treatments with organic matter showed the highest value, but this did not affect the yield negatively. For the persistence variable, it was possible to determine that in the control treatment there was a decrease in the amount of germinating spores with the advance in time and that the treatments applied caused variable responses regarding this behavior, showing equations with different slopes and very different coefficients of variation. The treatment with MM presented for the second evaluation the lowest average amount of spores and the lowest radical severity index. Conclusion. The organic matter improved the yield of the plants and the use of MM reduced the severity and showed potential to reduce the presence of spores in the soil.

y de la mostaza en particular es la hernia de las crucíferas, causada por el protista biotrofo obligado Plasmodiophora brassicae (Wor). Este microorganismo se ha dispersado por todo el mundo, posee una gran cantidad de plantas hospederas, aproximadamente 330 géneros y 3700 especies de la familia Brassicaceae (Dixon 2009) y se estima que puede reducir el rendimiento hasta en un $50 \%$ y causar un $60 \%$ de pérdida de cultivo (Strehlow et al. 2015). En ocasiones es necesario dejar de sembrar cultivos susceptibles 
o bien abandonar los lotes con presencia de altas poblaciones de este microorganismo.

El ciclo de $P$. brassicae se divide en 2 fases; en la primera, los exudados de la planta (y otras sustancias) inducen la germinación de las estructuras de resistencia y estas producen las zoosporas que penetran los pelos radicales. A esto se le atribuye la hiperplasia e hipertrofia celular, que induce el crecimiento anormal de las raíces (Riascos et al. 2011), así como la disminución en la absorción y el transporte de agua y nutrientes (Dixon 2014). En la segunda fase, la enfermedad llega hasta el córtex de la raíz, el plasmodio se divide en estructuras multinucleadas y se fracciona formando nuevas estructuras de resistencia que luego son liberadas al suelo (Riascos et al. 2011). Estas estructuras de resistencia o esporas pueden vivir sin la presencia de un hospedero susceptible hasta por 2 décadas, aunque el promedio de persistencia es de alrededor de 4 años (Friberg et al. 2008, Dixon 2009).

Björling (2013) señala que el éxito de $P$. brassicae se debe a que sus estructuras de reproducción o esporas están bien protegidas, por lo que el microorganismo tiene la capacidad de sobrevivir en el suelo bajo condiciones adversas, en tanto que, dichas estructuras responden germinando rápidamente una vez que encuentran un hospedero compatible.

El combate químico de enfermedades, como la hernia de las crucíferas, ha sido realizado en su mayoría mediante agroquímicos como PCNB, Clorotalonil, Triclamida, Fluazinam, Flusufamida, Carbendazim, Quintozeno, cyazofamida (Tanaka et al. 1999, Wang et al. 2017). Sin embargo, el control del $P$. brassicae no ha sido exitoso y el elevado uso de agroquímicos ha provocado daños al suelo y al ambiente, como contaminación, desequilibrio en las poblaciones microbiológicas, resistencia y otros (Castillo y Guerrero 2008, Sánchez-Bayo y Tennekes 2017).

Por tanto, se hace necesaria la implementación de nuevas técnicas favorables para el ambiente y que reduzcan no solo los costos de producción, sino también los riesgos de la emergencia de nuevos causales de enfermedades, producto de las técnicas implementadas en los sistemas agrícolas convencionales. Estas nuevas estrategias han estado orientadas hacia la incorporación de materia orgánica al suelo y el uso de microorganismos con capacidad biocontroladora.

La presencia de materia orgánica es fundamental para la salud y calidad del suelo, ya que modifica y mejora las condiciones decaídas del mismo. La materia orgánica aporta nutrientes a los diversos organismos, incluyendo las plantas; además, la diversidad microbiana y el sustrato energético, para el componente microbiano que implica, deriva en la generación de procesos afines a la salud del suelo y de las plantas (Kibblewhite et al. 2008).

Los abonos orgánicos ejercen su capacidad supresora mediante la sinergia con los microorganismos antagonistas, la carga microbiana que aumenta la competencia por recursos, la liberación de compuestos tóxicos, durante la descomposición de la materia orgánica, la inducción de la respuesta sistémica en la planta hospedera, entre otras causas (Lockwood 1988, Buysens et al. 1996, Zhang et al. 1996, Bonanomi et al. 2018, Neher 2019). Niwa et al. (2007) encontraron efectos importantes en la supresión de $P$. Brassicae, debido a las incorporaciones de materia orgánica.

Con respecto a los microorganismos biocontroladores, Auer y Ludwig-Müller (2015) indican que la colonización de las raíces por parte de este tipo de microorganismos puede promover el crecimiento radical y la defensa de las plantas mediante la mejora de las respuestas inmunológicas.

Las bacterias de los géneros Pseudomonas y Bacillus son conocidas como las bacterias más eficientes para el control de microorganismos, causales de enfermedades presentes en la parte foliar y en la raíz, y se ha demostrado el control de $P$. brassicae mediante el uso de dichas bacterias (Einhorn et al. 1991), así como también la reducción en la presencia de síntomas (Yeoung et al. 2003). En el género Bacillus se han observado mecanismos, tales como la competencia por 
sustrato y fuente de alimentación, la producción de antibióticos, enzimas y de otras sustancias, como sideróforos, los cuales se han relacionado con la actividad biocontroladora (Bais et al. 2006). Además, se conoce el potencial que tiene este microorganismo para solubilizar fosfatos, fijar nitrógeno y promover el crecimiento vegetal (Lugtenberg y Kamilova 2009).

Referente al género Pseudomonas, este es reconocido como controlador biológico, mediante la protección a semillas y raíces. En diversos estudios se ha encontrado que bacterias de este género producen sustancias antifúngicas y antibióticas que han controlado enfermedades causadas por Fusarium spp. y Gaeumannomyces spp. (Imran et al. 2006). Además, se ha observado que Pseudomonas incrementa la resistencia sistémica de las plantas debido al incremento en la actividad de quitinasas y peroxidasas (Nandakumar et al. 2001).

Otra opción, dentro del control biológico, es el uso de los microorganismos de montaña (MM), que se define como un "consorcio microbiano" o inoculo microbiano "complejo", de elaboración artesanal y con potencial para la expresión de diversidad de microorganismos y potencialidad de interacciones y procesos. Esta opción es utilizada por los productores agrícolas ecológicos, con el fin de incorporar esa diversidad a sistemas menos diversos o decaídos (Castro et al. 2015). Los MM son un cultivo mixto de microorganismos benéficos naturales, que no han recibido manipulación genética, son fisiológicamente compatibles y se encuentran en los ecosistemas naturales, específicamente las zonas boscosas. Dentro de los componentes microbianos de los MM se encuentran: actinomicetos, hongos, bacterias fototróficas y lácticas, levaduras, solubilizadores de $\mathrm{P}$, fijadores de $\mathrm{N}$, entre otros (Castillo y Guerrero 2008, Castro et al. 2015). Estos constituyen un inóculo microbiano que puede mejorar las condiciones del suelo y las plantas mediante la diversidad de funciones particulares y aquellas emergentes de las relaciones entre sus componentes (Castro et al. 2015).

La presente investigación tuvo como objetivo evaluar el potencial del abono orgánico, los microorganismos de montaña (MM) y el producto biológico comercial Fertibiol, como métodos alternativos para el control biológico de la hernia de las crucíferas (Plasmodiophora brassicae wor.) en el cultivo de mostaza china (Brassica rapa sp. pekinensis).

\section{MATERIALES Y MÉTODOS}

\section{Sitio experimental}

La fase experimental se llevó a cabo en el Invernadero del Departamento de Microbiología Agrícola del Centro de Investigaciones Agronómicas (CIA) de la Universidad de Costa Rica, ubicado en el cantón de Montes de Oca, San José, Costa Rica.

\section{Material experimental}

Suelo: para la prueba se utilizó suelo con presencia natural del patógeno que se obtuvo de una finca ubicada en Tapezco de Zarcero, Alajuela a $1821 \mathrm{msnm}$, en las coordenadas: latitud: $10^{\circ} 13^{\prime} 53^{\prime \prime} \mathrm{N}$ y longitud: $84^{\circ} 23^{\prime} 40^{\prime \prime} \mathrm{O}$. Se identificó el patógeno mediante la observación de síntomas como hernias de gran tamaño y marchitez en los cultivos presentes: brócoli, coliflor y repollo, simultáneamente. Se determinó la cantidad de ADN en el suelo y el suelo + abono y la cantidad de esporas de $P$. brassicae, valores que corresponden a $27,9 \mathrm{ng} . \mu \mathrm{l}^{-1}\left(6,54 \times 10^{9}\right.$ esporas $)$ y 25,7 ng. $\mu l^{-1}\left(6,39 \times 10^{8}\right.$ esporas) respectivamente.

Las características químicas del suelo utilizado se muestran en la Tabla 1. 
Tabla 1. Condición química del suelo de Tapezco, Zarcero.

\begin{tabular}{|c|c|c|c|c|c|c|c|c|c|c|c|c|}
\hline & \multirow{2}{*}{$\begin{array}{l}\mathrm{pH} \\
\mathrm{H}_{2} 0\end{array}$} & \multirow{2}{*}{$\begin{array}{l}\text { Acidez } \\
\text { Acidez }\end{array}$} & \multicolumn{4}{|c|}{$\mathrm{cmol}(+) \cdot \mathrm{L}^{-1}$} & \multirow{2}{*}{$\begin{array}{l}\% \\
\text { SA }\end{array}$} & \multicolumn{5}{|l|}{$M g . L^{-1}$} \\
\hline & & & $\mathrm{Ca}$ & $\mathrm{Mg}$ & K & CICE & & $\mathrm{P}$ & $\mathrm{Zn}$ & $\mathrm{Cu}$ & $\mathrm{Fe}$ & $\mathrm{Mn}$ \\
\hline $\mathrm{NC}$ & 5,5 & 0,5 & 4 & 1 & 0,2 & 5 & 10 & 10 & 3 & 2 & 10 & 5 \\
\hline Suelo & 5,5 & 0,22 & 8,42 & 0,94 & 0,54 & 10,12 & 2 & 52 & 8,6 & 10 & 96 & 10 \\
\hline & \multicolumn{2}{|c|}{$\% \mathrm{C}$} & \multicolumn{2}{|l|}{$\% \mathrm{~N}$} & $\% \mathrm{MO}$ & \multicolumn{2}{|c|}{$\mathrm{Ca} \cdot \mathrm{Mg}^{-1}$} & \multicolumn{2}{|l|}{$\mathrm{Ca} \cdot \mathrm{K}^{-1}$} & $\mathrm{Mg} . \mathrm{K}^{-1}$ & \multicolumn{2}{|c|}{$\mathrm{Ca}+\mathrm{Mg} . \mathrm{K}^{-1}$} \\
\hline $\mathrm{NC}$ & & & & & & \multicolumn{2}{|c|}{$2-5$} & \multicolumn{2}{|l|}{$5-25$} & $2,5-15$ & \multicolumn{2}{|c|}{$10-40$} \\
\hline Suelo & \multicolumn{2}{|c|}{7,10} & \multicolumn{2}{|l|}{0,70} & 10,1 & \multicolumn{2}{|c|}{8,95} & \multicolumn{2}{|l|}{15,59} & 1,74 & \multicolumn{2}{|c|}{17,33} \\
\hline
\end{tabular}

Material vegetal: se utilizó mostaza china Taranko F1, obtenido en forma de almácigo en la empresa Villaplant.

Microorganismos de montaña (MM): el mantillo de bosque, para la elaboración del MM sólido, fue recolectado de una zona boscosa a 1730 msnm, ubicado en las coordenadas: latitud 958'60' y longitud $83^{\circ} 57^{\prime} 60^{\prime}$ '. Las características del MM líquido 12 días después de la activación se describen en la Tabla 2.

Tabla 2. Características del MM líquido 12 días después de la activación líquida.

\begin{tabular}{|c|c|}
\hline $\mathrm{pH}$ & 3,7 \\
\hline $\mathrm{CE}\left(\mathrm{mS} . \mathrm{cm}^{-1}\right)$ & 7,1 \\
\hline Lactobacilos ufc. $\mathrm{ml}^{-1}$ & $1,5^{*} 10^{9}$ \\
\hline Actinomicetes ufc.ml $\mathrm{l}^{-1}$ & $1,0 * 10^{6}$ \\
\hline Bacterias ufc.ml $\mathrm{l}^{-1}$ & $1,2 * 10^{8}$ \\
\hline Levaduras ufc. $\mathrm{ml}^{-1}$ & $3,3 * 10^{8}$ \\
\hline Solubilizadores de $\mathrm{P}$ nmp. $\mathrm{ml}^{-1}$ & $1,1 * 10^{7}$ \\
\hline Fijadores de $\mathrm{N}$ ufc. $\mathrm{ml}^{-1}$ & $4,7 * 10^{8}$ \\
\hline
\end{tabular}

Abono orgánico: el abono orgánico utilizado fue tipo Bokashi, elaborado en la Finca Guadalupe en Zarcero. Según el análisis micro-

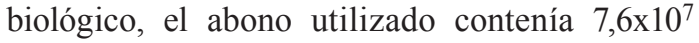


micetos y $1,4 \times 10^{6}$ UFC. $g^{-1}$ Hongos. Por su parte, el análisis químico indicó que el abono contenía una humedad de 50\%, pH de 6,6, CE 21,3, \%C: 21,02 y relación $\mathrm{C} / \mathrm{N}$ : 11,0 y los nutrientes se encontraron en las siguientes cantidades: N: 1,92, P: 0,46, Ca: 3,90, Mg: 0,42, K: 1,91, S: 1,61, Fe: 4936, Cu: 63, Zn: 174, Mn: 383 y B: 14.

Fertibiol: es un producto comercial elaborado en el Centro de Investigaciones Agronómicas (CIA) de la Universidad de Costa Rica. El mismo contiene cepas de Bacillus y Pseudomonas aisladas en Costa Rica.

Macetas: el tamaño de las macetas fue de 1,5 L y al momento de la siembra se agregó $1 \mathrm{~L}$ de suelo por maceta.

\section{Diseño del experimento}

Se establecieron 7 tratamientos con 5 repeticiones cada uno y con 6 plantas cada repetición para un total de 210 macetas. Los tratamientos evaluados fueron: Testigo con plantas (TP); Testigo sin plantas (TSP); Microorganismos de Montaña (MM); Microorganismos de Montaña + Abono orgánico ( $\mathrm{MM}+\mathrm{AO})$; Abono orgánico (AO); Fertibiol (FB); Fertibiol + Abono orgánico $(\mathrm{FB}+\mathrm{AO})$.

\section{Aplicación de los tratamientos}

Previo a la siembra se tomaron muestras de aprox. $600 \mathrm{ml}$ de suelo para realizar análisis microbiológico, químico y rtPCR. En los tratamientos con abono orgánico se realizó la mezcla de $1 \mathrm{~L}$ de suelo con $15 \% \mathrm{v} / \mathrm{v}$ de abono orgánico (se ha observado en otros ensayos que los mejores resultados en crecimiento de las plantas, y otras 
variables, se logran con aplicación entre el 20 y el $25 \%$ de abono orgánico) y se procedió al llenado de las macetas. Los demás tratamientos llevaban únicamente suelo.

Posteriormente, para los tratamientos Fertibiol y MM se realizó una dilución en agua al 10\% (100 ml de producto en $900 \mathrm{ml}$ de agua, según se recomienda) cada uno y en recipientes separados. Las plántulas se sumergieron en las diluciones, según tratamiento, por un período de 1 min. Luego de la aplicación de los tratamientos, se realizó la siembra.

Se realizaron 2 ciclos de cultivo en el mismo suelo, al finalizar estos, se retiraron las plantas para ser evaluadas, para el segundo ciclo se mezcló el suelo de las repeticiones de un mismo procedimiento y se aplicaron los tratamientos antes de volver a sembrar.

Para el primer ciclo, se realizó la mezcla del abono orgánico al 15\% v/ y para el ciclo 2, se adicionó abono al $5 \% \mathrm{v} / \mathrm{v}$ previo a la siembra.

\section{Variables evaluadas}

Las evaluaciones fueron de tipo destructivo y las variables evaluadas fueron:

Peso fresco y peso seco: se determinó la acumulación de biomasa en las plantas de mostaza al final de cada ciclo de cultivo al separar la parte aérea y radical.
La evaluación se realizó de la siguiente forma: la parte radical se lavó con agua a presión para separar la mayor cantidad de suelo posible. Luego de ser pesadas las partes radicales y aéreas de cada planta y cada repetición, se colocaron en bolsas de papel. Posteriormente, para el peso seco, se dejaron las muestras $24 \mathrm{~h}$ en estufa a $65^{\circ} \mathrm{C}$ y luego se volvieron a pesar sin la bolsa.

\section{Severidad}

Paralelamente a la evaluación de peso fresco y peso seco, cuando se tenían las raíces lavadas, se procedió a evaluar el índice de severidad causado por el patógeno a nivel radical, se colocaron las partes radicales que corresponden a una misma repetición en una superficie para ser evaluadas según el índice promedio de severidad.

El índice promedio de severidad se evaluó en las raíces mediante una escala ya determinada por el Laboratorio de Fitopatología de la UCR (Figura 1), los datos fueron calculados con la siguiente fórmula:

$$
\mathrm{IPS}=\sum \boldsymbol{n}_{i} \boldsymbol{g}_{i / N G \times 100}
$$

Donde $\mathrm{n}_{\mathrm{i}}$ : número de unidades de la categoría $\mathrm{i}, \mathrm{g}_{\mathrm{i}}$ : el grado de la categoría i, N: número total de unidades evaluadas y G: grado máximo de la escala. 


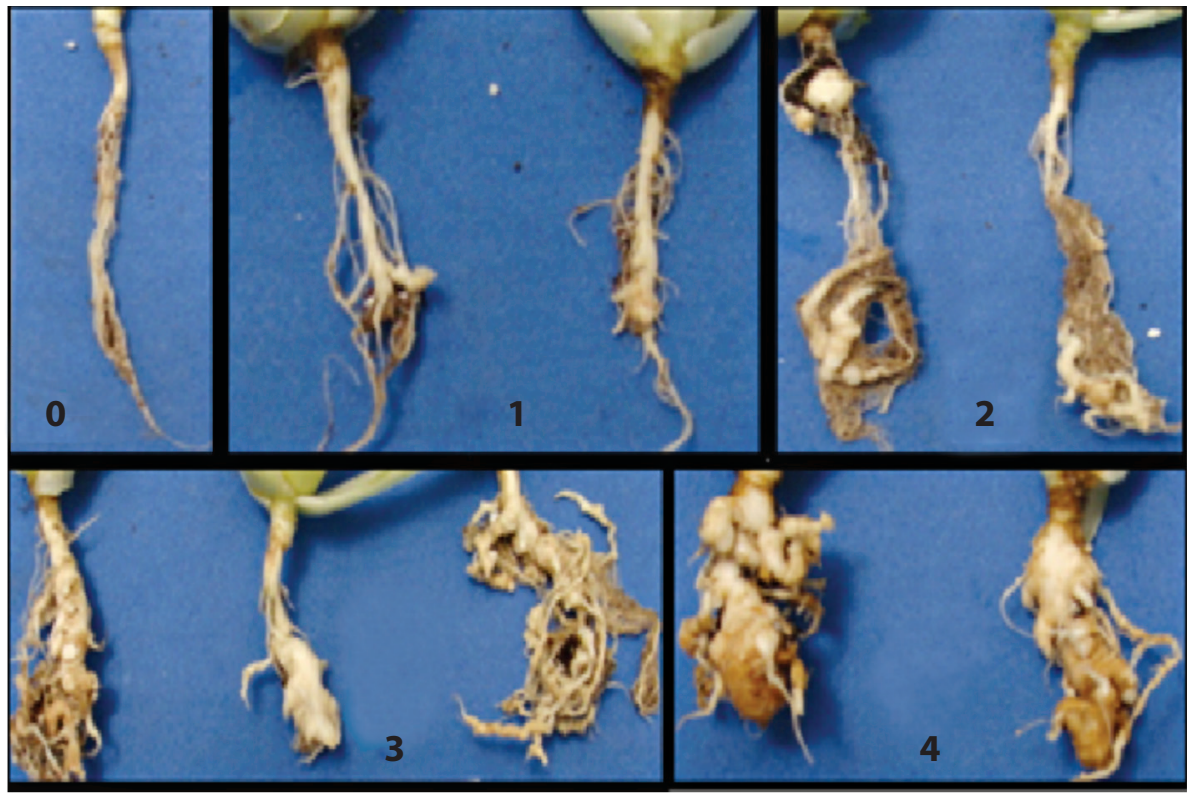

Figura 1. Escala para evaluación de severidad de Plasmodiophora brassicae.

Facilitada por el Laboratorio de Fitopatología, Universidad de Costa Rica, San José, Costa Rica.

Donde:

Nivel 0: no se observan agallas en las raíces de las plantas. La raíz se observa fibrosa y bien ramificada.

Nivel 1: muy pocas agallas y de un tamaño pequeño. Se observa una buena porción de raíz fibrosa y funcional.

Nivel 2: pocas agallas, pero con un nivel considerable de abultamiento. Se observa aún una buena porción de raíz fibrosa.

Nivel 3: se observa una cantidad relativamente alta de agallas, que si bien no son muy grandes, si presentan un nivel de abultamiento considerable. Estas se distribuyen en toda la raíz y hay una disminución de la porción fibrosa de la raíz.
Nivel 4: se observan agallas de gran tamaño que cubren casi la totalidad del sistema radical. Casi no se observa raíz fibrosa.

\section{Análisis de $P$. brassicae}

El análisis rtPCR se llevó a cabo en el Laboratorio de Técnicas Moleculares aplicadas a la Fitoprotección del CIPROC-UCR.

Extracción de ADN presente en el suelo: el ADN proveniente del suelo se extrajo con el kit Nucleospin Soil, Macherey Nagel. El ADN se cuantificó mediante un espectofotómetro BioPhotometer Plus 6132 (Eppendorf). El ADN del patógeno se midió en ng. $\mu \mathrm{l}^{-1}$ (nanogramos por microlitro) de ADN.

rtPCR: mediante la técnica de PCR en tiempo real, se cuantificó el ADN del patógeno $P$. brassicae presente en el suelo al final de cada ciclo de cultivo. Se tomaron 5 muestras en cada uno de los 6 tratamientos para un total de 30 
muestras en cada ciclo. La cantidad de ADN obtenida y cuantificada, mediante electroforesis, se amplificó con tiempo real utilizando los primers, según Deora et al. (2015). Se prepararon concentraciones seriadas del ADN, las cuales sirvieron para estimar la cantidad de ADN en las muestras analizadas provenientes de los suelos bajo tratamiento. Además, se realizó una curva a partir de un número específico de esporas en reposo, que se muestra en la Figura 2; dicha curva se puede utilizar para determinar las cantidades de esporas correspondientes a la lectura del rtPCR.

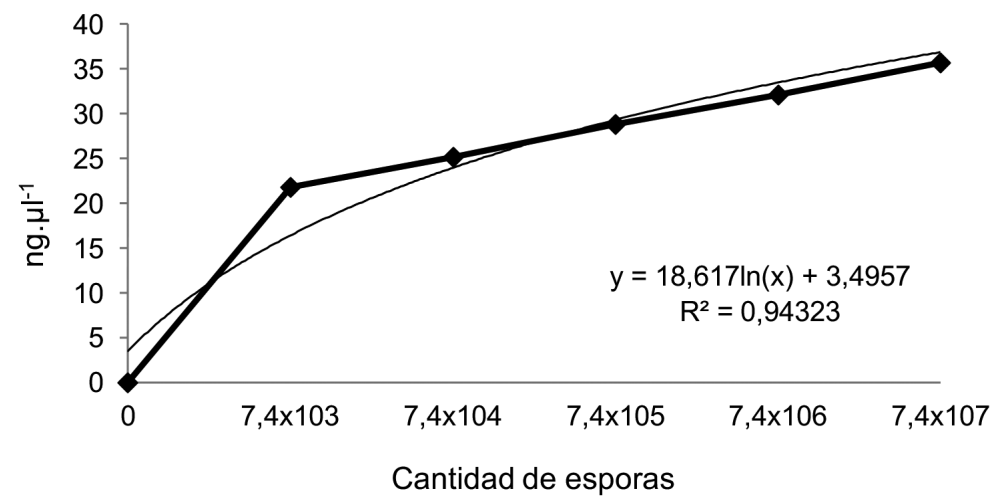

Figura 2. Curva para los valores de rtPCR elaborada a partir de un número determinado de esporas en reposo.

\section{Análisis estadístico}

Para las variables peso fresco y peso seco de la parte aérea y radical, se aplicó la prueba de Kruscal-Wallis, debido a que no presentaban una distribución normal y para determinar diferencias estadísticas entre los tratamientos se utilizaron las pruebas de Shapiro-Wilks y Levene.

A las variables Severidad y Relación R/T, se les realizó un análisis de varianza (ANDEVA) y la prueba DGC para la separación de medias. Por último, para la variable de rtPCR, se realizaron las pruebas de Shapiro-Wilks, Levene y Kruscal-Wallis.

\section{RESULTADOS}

\section{Peso fresco y peso seco}

En la Tabla 3, se presentan los datos obtenidos para la acumulación de biomasa fresca y seca de la parte aérea, de manera independiente para cada evaluación y un valor promedio total de las 2 evaluaciones. 
Tabla 3. Promedio del peso fresco y seco obtenido en la parte aérea de plantas de mostaza durante 2 ciclos de cultivo.

\begin{tabular}{lllllll}
\hline \multicolumn{3}{c}{ Peso fresco (gramos) } & \multicolumn{3}{c}{ Peso seco (gramos) } \\
\hline Trat. & $\begin{array}{l}\text { EV 1 } \\
\mathrm{p}=0,0045\end{array}$ & $\begin{array}{l}\text { EV 2 } \\
\mathrm{p}=0,0001\end{array}$ & Total & $\begin{array}{l}\text { EV 1 } \\
\mathrm{p}=0,0003\end{array}$ & $\begin{array}{l}\text { EV2 } \\
\mathrm{p}=0,0001\end{array}$ & Total \\
\hline TP & $81,26 \mathrm{ab}$ & $48,97 \mathrm{a}$ & 130,23 & $19,13 \mathrm{ab}$ & $17,85 \mathrm{a}$ & 36,98 \\
$\mathrm{AO}$ & $307,28 \mathrm{c}$ & $139,95 \mathrm{c}$ & 447,23 & $50,72 \mathrm{c}$ & $32,06 \mathrm{c}$ & 82,78 \\
MM & $64,97 \mathrm{a}$ & $48,13 \mathrm{a}$ & 113,10 & $19,53 \mathrm{a}$ & $17,71 \mathrm{a}$ & 37,24 \\
MM + AO & $232,97 \mathrm{bc}$ & $111,12 \mathrm{bc}$ & 344,09 & $47,99 \mathrm{bc}$ & $29,04 \mathrm{bc}$ & 77,03 \\
FB & $65,15 \mathrm{ab}$ & $54,86 \mathrm{ab}$ & 120,01 & $19,29 \mathrm{a}$ & $18,16 \mathrm{ab}$ & 37,45 \\
FB + AO & $238,41 \mathrm{c}$ & $94,57 \mathrm{bc}$ & 332,98 & $49,69 \mathrm{bc}$ & $28,84 \mathrm{bc}$ & 78,53 \\
\hline
\end{tabular}

Letras distintas indican diferencias significativas $(\mathrm{p} \leq 0,05)$.

Los mayores valores en el peso fresco aéreo para ambas evaluaciones se obtuvieron en los 3 tratamientos que contenían abono orgánico (Tabla $3)$, los cuales resultaron estadísticamente similares. La acumulación de biomasa fresca aérea fue mayor en el tratamiento $\mathrm{AO}$ con un valor acumulado de 447,23 g, mientras que los tratamientos $\mathrm{FB}+\mathrm{AO}$ y $\mathrm{MM}+\mathrm{AO}$ obtuvieron 344,09 g y 332,98 g, respectivamente. Los demás tratamientos sin abono orgánico (TP, MM y FB) fueron similares estadísticamente y obtuvieron pesos menores a $130 \mathrm{~g}$.

Respecto al peso seco aéreo, la mayor acumulación de biomasa seca se encontró en estos mismos tratamientos; con valores de $82,78 \mathrm{~g}$ para $\mathrm{AO}, 78,53$ g para $\mathrm{FB}+\mathrm{AO}, \mathrm{y} 77,03$ g para $\mathrm{MM}+$ AO. Los tratamientos MM y FB se comportaron similares al testigo al obtener los valores más bajos alrededor de $19 \mathrm{~g}$ (Tabla 3).

En los pesos de masa fresca y masa seca de la parte radical, se obtuvieron también valores mayores para los tratamientos $\mathrm{AO}, \mathrm{MM}+\mathrm{AO}$ y $\mathrm{FB}+\mathrm{AO}$ y fueron iguales estadísticamente. Nuevamente los tratamientos sin abono orgánico mostraron los pesos más bajos. Los tratamientos MM y Fertibiol obtuvieron resultados similares al testigo, dejando en evidencia que no representaron, en este caso, un aporte significativo en el crecimiento de las plantas de mostaza.

Los resultados obtenidos mostraron un comportamiento claro de mayor acumulación de biomasa fresca y seca en los tratamientos que contenían abono orgánico, así como el efecto benéfico sobre el crecimiento de las plantas de mostaza (Tabla 3). Sobre este tema, autores como Ruíz et al. (2007), definen que hay una relación positiva entre la aplicación de abonos orgánicos y el desarrollo de los cultivos. El abono orgánico realiza un aporte a la nutrición de las plantas, ya sea de manera directa o porque por medio de la adición de nutrientes y de un inoculo microbiano se promueve el crecimiento vegetal o se incrementan procesos como la mineralización, solubilización y absorción, que pone en disponibilidad los nutrientes, lo cual aumenta el rendimiento, crecimiento y calidad de los cultivos (Orozco y Muñoz 2012, Durango 2014, Bonanomi et al. 2018, Neher 2019). De acuerdo con la literatura, suelos que tienen materia orgánica en abundancia son fértiles, tienen estructura y son más productivos (Bittner et al. 2007, Garro 2016). Por otro lado, la materia orgánica aporta ácidos húmicos, reguladores de crecimiento y hormonas (Valverde et al. 2011). Dichas sustancias pueden contribuir a un mayor crecimiento y rendimiento de las plantas, lo cual da como resultado mayor acumulación de biomasa aérea y radical, gracias al aporte de nutrientes, carbono y energía; se reporta en la literatura que estos compuestos influyen positivamente en la respiración y el ciclo de Krebs al aumentar la producción de ATP (Moreno et al. 2014, Reyes et al. 2016, Arteaga et al. 2006). 
Por otra parte, los datos muestran la tendencia a un efecto negativo de la incorporación de los productos MM y Fertibiol en mezcla con el abono orgánico (Figura 3), ya que se da una disminución en la acumulación de biomasa fresca. Esta disminución puede deberse a que ambos productos presentan un valor de $\mathrm{pH}$ bajo, alrededor de 4. De esta manera, la forma de aplicación de dichos productos, que fue por inmersión de la parte radical directamente en la solución, pudo tener un efecto directo de daño sobre las raíces, o modificar las relaciones biológicas en la rizosfera $y$, debido a que existe una interdependencia entre el crecimiento radical y aéreo (Willigen y Noordwijk 1987), este último también se vio afectado. Por otro lado, Neuhauser y Fargione (2004) mencionan que los microorganismos se ubican en la rizosfera, debido a que es ahí donde se encuentran la mayor cantidad de nutrientes y exudados de las plantas y es posible que se presenten relaciones sinérgicas o de competencia física por nutrientes o bien otros tipos de antagonismo (Cano 2011).


Figura 3. Relación raíz/tallo (dato sobre el eje de las X) para el peso fresco de plantas de mostaza durante la primera A y segunda $B$ evaluación. Valor de $\mathrm{p}=0,0001$ y $\mathrm{p}=0,0005$ respectivamente. Letras distintas indican diferencias significativas $(\mathrm{p} \leq 0,05)$ en cada columna. 


\section{Relación raíz/tallo}

Como se observa en la Figura 3A, para la primera evaluación, la relación raíz/tallo en el tratamiento $\mathrm{AO}$ muestra el valor estadísticamente más bajo $(0,22)$, mientras que los otros 2 tratamientos con abono orgánico $(\mathrm{MM}+\mathrm{AO}$ y $\mathrm{FB}+\mathrm{AO})$ obtuvieron los valores estadísticamente más altos, de 0,38 y 0,37 respectivamente. Para esta variable, se hacen significativas las tendencias observadas al evaluar el peso seco y fresco en el tratamiento $\mathrm{AO}$, de tal manera que la aplicación tanto de MM como de FB, junto con el AO, disminuyeron los valores del peso fresco aéreo, mientras que aumentaron los de la raíz.

Los tratamientos FB y MM fueron estadísticamente iguales al testigo TP (Figura 3A) respecto a la relación raíz/tallo, de lo que se deduce que la aplicación de MM o FB, de manera individual (sin $\mathrm{AO}$ ), no causó ningún efecto que fuera estadísticamente significativo sobre la relación $\mathrm{R} / \mathrm{T}$ bajo las circunstancias de la evaluación.

Para la segunda evaluación (Figura 3B), el análisis de los datos para la relación $\mathrm{R} / \mathrm{T}$ genera únicamente 2 grupos. En los tratamientos con aplicación de abono orgánico $(\mathrm{AO}, \mathrm{MM}+\mathrm{AO}$ y $\mathrm{FB}+\mathrm{AO}$ ) se obtuvo las relaciones más bajas con valores entre 0,26 y 0,29 , lo cual coincide con una mayor ganancia de biomasa aérea y radical, mientras que con los tratamientos sin abono orgánico se obtuvo una relación más altas entre 0,35 y 0,38 .

La relación $\mathrm{R} / \mathrm{T}$ para el peso seco en la evaluación 1 y 2 (Figura 4) muestra que los tratamientos TP, MM y FB obtuvieron las relaciones más elevadas entre 0,54 y 0,56 . Los tratamientos $\mathrm{AO}, \mathrm{FB}+\mathrm{AO}$ y $\mathrm{MM}+\mathrm{AO}$ no resultaron estadísticamente diferentes y obtuvieron los valores más bajos en las relaciones $\mathrm{R} / \mathrm{T}$; en este caso el tratamiento con la aplicación de solamente $\mathrm{AO}$ en la evaluación 1 no se diferenció de $\mathrm{FB}+\mathrm{AO}$ y $\mathrm{MM}+\mathrm{AO}$, lo que parece indicar que la diferencia encontrada para esta misma variable, pero para los pesos frescos, estuvo relacionada con la turgencia de las plantas, dato que es relevante para la comercialización de la mostaza. Ya que la parte aérea fresca de la mostaza es la que es comercializable, se puede deducir que los menores valores de la relación $\mathrm{R} / \mathrm{T}$ indican las mejores cualidades comerciales de las plantas. Para esta investigación, el menor dato obtenido para R/T fue de 0,22 para el tratamiento con solo aplicación de abono orgánico, que correspondió a las plantas que acumularon mayor biomasa aérea fresca. 



Figura 4. Relación raíz/tallo (datos sobre el eje de las X) para el peso seco de plantas de mostaza durante la primera A y segunda $\mathrm{B}$ evaluación. Valor de $\mathrm{p}=<0,0001$ para ambas evaluaciones. Letras distintas indican diferencias significativas $(\mathrm{p} \leq 0,05)$ en cada columna.

Según (Barrios et al. 2014), existe una interdependencia entre la acumulación de biomasa radical y aérea, que permite un equilibrio en el crecimiento de las plantas. Villalobos (2001) indica que una correcta relación raíz/tallo le permite a las plantas ajustarse a la cantidad de nutrientes absorbidos a nivel radical y a los productos de la fotosíntesis. Las plantas tienen la capacidad de adecuarse a la cantidad de minerales y carbono, así que cuando se encuentran en cantidades adecuadas de nutrientes requieren menor sistema radical para mantener el follaje y esto provoca una disminución en la relación raíz/tallo.
Es importante destacar que el tratamiento en el que se adicionó abono orgánico no solamente obtuvo datos mayores para las variables de peso fresco y peso seco a nivel foliar y radical, sino relaciones $\mathrm{R} / \mathrm{T}$ menores, por tanto es posible deducir un efecto positivo que de mantenerse en condiciones de campo podría incrementar sustancialmente la productividad del cultivo.

Otra información importante de rescatar es que se observó una mayor producción de raíces secundarias en los tratamientos con adición de abono orgánico, aunque no fueron cuantificadas, el comportamiento se puede observar en 
la Figura 5. Al respecto, Reyes y Valery (2015) mencionan la ventaja que le confiere al cultivo la incorporación de abono orgánico, ya que el sistema radical incrementa el crecimiento tanto de raíces laterales como de pelos absorbentes y provoca que los procesos bioquímicos se aceleren promoviendo, de esta manera, el crecimiento y la activación de los mecanismos de defensa de las plantas. Además, el abono orgánico favorece el incremento de las poblaciones de microorganismos con diferentes funciones en el control biológico, como el antagonismo, la competencia y la producción de sustancias antibióticas y antifúngicas (Garro 2016). Dichas poblaciones se encuentran dentro de un ambiente dinámico donde se dan interacciones entre microorganismos, que son complejas, y se crean comunidades o consorcios que dependen de las condiciones físicas y químicas del suelo en general y de la rizosfera en particular. Dentro de los microorganismos, se encuentran diversos hongos y bacterias que se asocian con las raíces del cultivo; en esta zona se produce una gran cantidad de sustancias con potencial para la estimulación del crecimiento y control de enfermedades (Cano 2011).
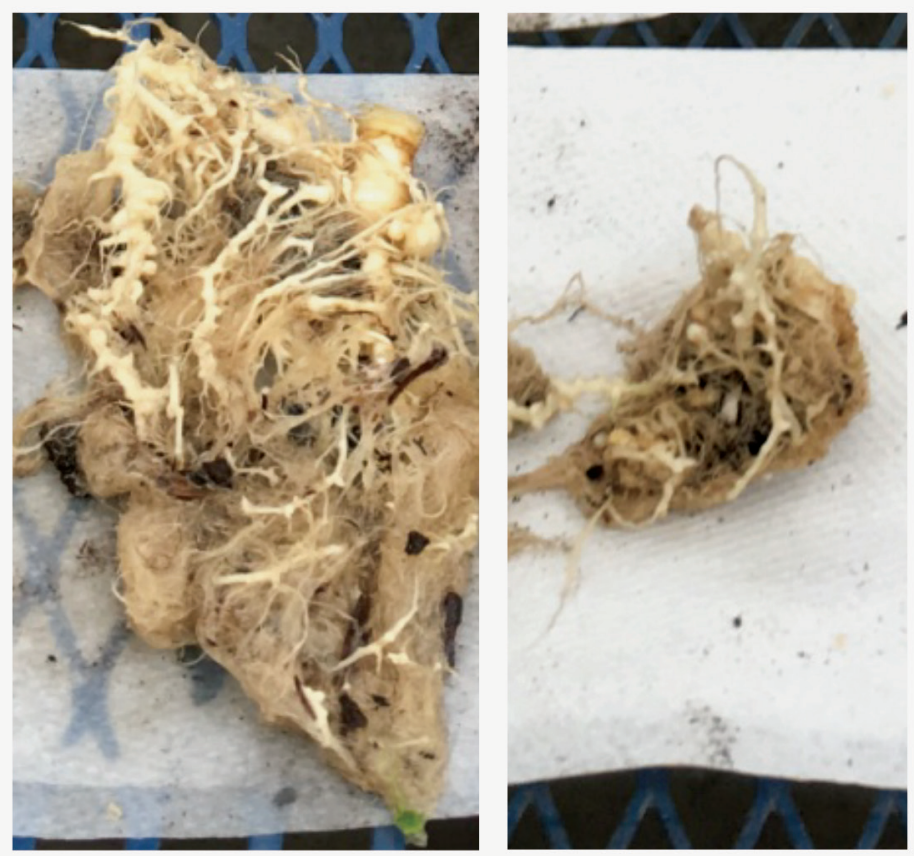

Figura 5. Producción de raíces secundarias en el sistema radical. Izquierda, sistema radical promedio para los tratamientos con materia orgánica. Derecha, sistema radical promedio del tratamiento testigo.

\section{Severidad}

Respecto al índice promedio de severidad en la parte radical (Figura 6A), para la primera evaluación el valor fue menor para el tratamiento $\mathrm{MM}$ con $53 \%$, seguido por el TP
$(64 \%)$ sin existir diferencia estadística entre los 2. Todos los demás tratamientos obtuvieron valores estadísticamente mayores y sin diferencias entre ellos, el tratamiento $\mathrm{MM}+\mathrm{AO}$ fue el que mostró el mayor valor (83\%) en números absolutos. 


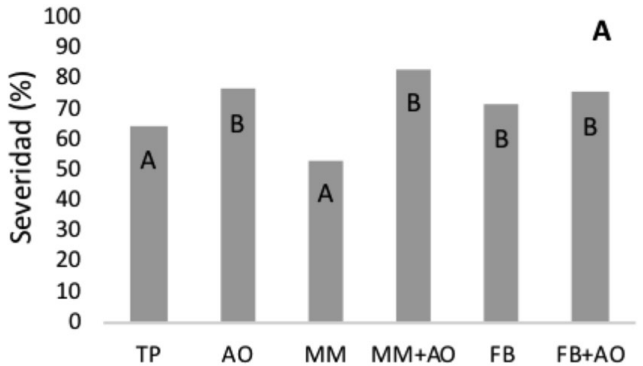

Tratamientos

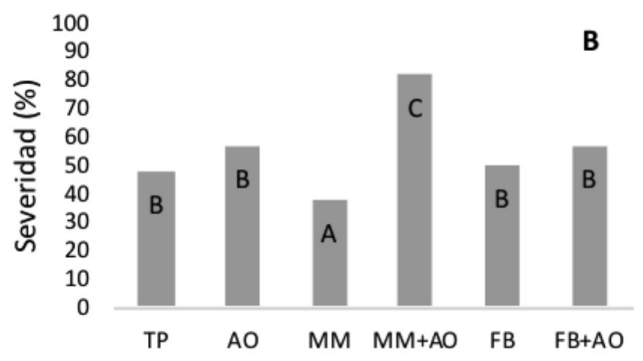

Tratamientos

Figura 6. Severidad del daño causado por el patógeno P. brassicae en plantas de mostaza durante los 2 ciclos de cultivo. Valores de $\mathrm{p}$ en evaluaciones 1 y 2 son: 0,0278 y $<0,0001$ respectivamente. Letras distintas en cada columna indican diferencias significativas $(\mathrm{p} \leq 0,05)$.

Para los tratamientos con abono orgánico esto resulta contrastante, ya que los resultados en cuanto al peso fresco y peso seco para estos mismos tratamientos mostraron los mayores valores en la acumulación de biomasa. Es decir, una alta presencia de agallas en la raíz no fue, en este caso, causal de un menor crecimiento, así las plantas en presencia de abono orgánico lograron los mayores pesos frescos y secos, mientras mostraron también el mayor índice de severidad.

Este comportamiento puede parecer contradictorio, ya que según la norma, las plantas que crecen libres de organismos causales de enfermedades pueden expresar mejor su potencial y desarrollarse correctamente. En este caso se evidencia el efecto benéfico por parte del abono orgánico, que permite a las plantas sobrellevar la presencia del agente causal. Se deduce de este hecho, las implicaciones económicas de mantener cantidades adecuadas de materia orgánica en los suelos, tanto en el aspecto de producción como en la manifestación de la sanidad de las plantas. Al respecto, Dixon (2009) indica que la adición de material orgánico por largos periodos de tiempo pueden lograr que suelos conductivos a Plasmodiophora brassicae se trasformen en suelos supresivos. Es importante destacar que esta materia orgánica debe encontrarse en su fase dinámica y que dicho dinamismo es gestionado por la función de la micro y macrobiota (Schofield et al. 2018).

En la segunda evaluación (Figura 6B), el tratamiento con MM mostró el menor valor de severidad, diferenciándose estadísticamente de todos los tratamientos incluyendo del TP; los tratamientos $\mathrm{TP}, \mathrm{AO}, \mathrm{FB}, \mathrm{FB}+\mathrm{AO}$ mostraron valores medios de severidad y fueron estadísticamente iguales. Para el tratamiento $\mathrm{MM}+\mathrm{AO}$, se obtuvo el mayor valor de severidad, este comportamiento fue tendencia para la primera evaluación. Al parecer bajo las condiciones específicas de la presente investigación, los factores MM y abono orgánico cuando se utilizaron en conjunto mostraron una causalidad positiva cuando se evaluó la variable severidad, dicho efecto no se presenta con el Fertibiol, lo que se puede constatar al observar que los tratamientos $\mathrm{AO}$ y $\mathrm{AO}+\mathrm{FB}$ son estadísticamente similares. Las condiciones particulares producto de la combinación del $\mathrm{AO}$ y el MM incrementaron el índice de agallamiento de las raíces y requieren una mayor investigación.

\section{Persistencia de $P$. brassicae: Análisis rtPCR}

En cuanto a la persistencia de esporas de $P$. brassicae en el suelo, para la primera evaluación (Figura 7A), los contenidos de $\mathrm{ADN}$ en ng. $\mu \mathrm{l}^{-1}$ fueron estadísticamente menores para los 
3 tratamientos con presencia de abono orgáni$\mathrm{co}$, en este caso los tratamientos $\mathrm{AO}, \mathrm{MM}+\mathrm{AO}$ Y FB+AO obtuvieron valores de 31,$11 ; 30,78$ y 32,14 respectivamente, los demás tratamientos presentaron valores mayores y estadísticamente iguales entre ellos, esto se puede explicar por el factor dilución debido a la aplicación de $15 \%$ de abono orgánico. Sin embargo, este comportamiento es contrario a lo encontrado cuando se evaluó la severidad en la primera evaluación, donde los tratamientos $\mathrm{AO}, \mathrm{MM}+\mathrm{AO}$ y $\mathrm{FB}+\mathrm{AO}$ fueron los que presentaron porcentajes más altos de severidad. Es importante tomar en cuenta en este caso que el abono orgánico proporciona sustancias, nutrientes y otros compuestos, no solo al cultivo, sino a todo el sistema compuesto en este caso por la unidad experimental y que incluye al agente causal Plasmodiophora brassicae; estas condiciones, por tanto, podrían incrementar el agallamiento en las raíces.

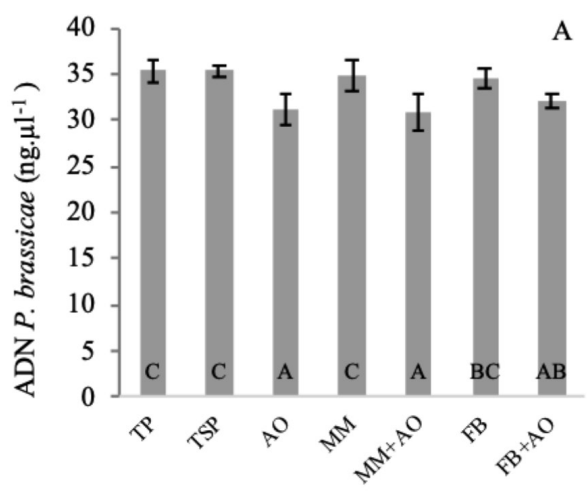

Tratamientos

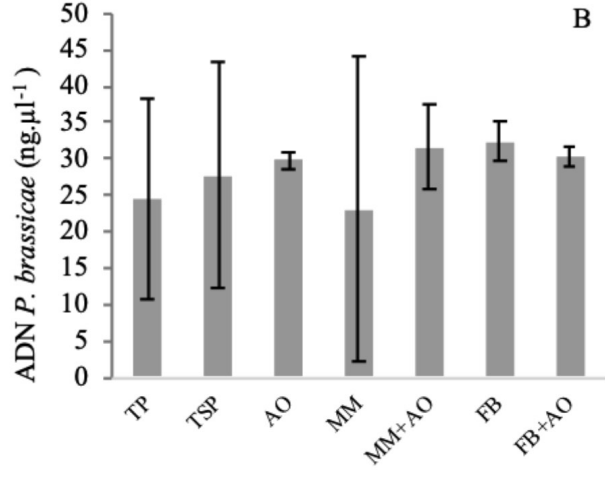

Tratamientos

Figura 7. Valores de rtPCR en suelo al finalizar el primer A y segundo B ciclo de cultivo de mostaza. 2018. Valor de $p=0,0007$ y 0,6473 respectivamente. Letras distintas indican diferencias significativas $(p \leq 0,05)$ en cada columna. San Pedro, San José.

Al realizar la segunda evaluación (Figura 7B), no se encontraron diferencias estadísticas significativas entre los tratamientos, debido, posiblemente, a que los tratamientos TP, TSP y MM presentaron CV altos (13,71; 15,57 y 21,04 respectivamente). Particularmente alto fue el del tratamiento con MM, que a su vez fue el que presentó la media menor, donde algunas repeticiones presentaron valores altos, mientras que prácticamente no se detectó la presencia de ADN en otras. Esto puede ser apreciado en las barras de error donde se muestra el coeficiente de variación (Figura 7B), de ello se puede deducir la alta complejidad de las relaciones que provoca la inoculación con MM y, por tanto, la incertidumbre que conlleva, ya que como lo ha establecido la física contemporánea, desviaciones iniciales muy débiles, entrañan enormes divergencias. Sin embargo, en términos prácticos el hecho de haber obtenido unidades experimentales, sin casi presencia de esporas de Plasmodiophora brassicae, puede tener implicaciones útiles importantes a nivel de campo. Por otro lado, la menor presencia promedio de $\mathrm{ADN}$ en el tratamiento $\mathrm{MM}$ se relaciona con el menor porcentaje de severidad obtenido en este mismo ciclo de cultivo.

Es relevante indicar que los coeficientes de variación para los tratamientos $\mathrm{AO}, \mathrm{MM}+\mathrm{AO}$, FB y FB+AO fueron muy bajos 1,$17 ; 5,81$ y 1,22 y similares entre sí, por lo que se puede concluir que la variación mencionada es una respuesta debida a los tratamientos y no a otros factores experimentales. 
En la Figura 8, se muestra el comportamiento de los valores de las evaluaciones de rtPCR al final de la etapa experimental de ambos ciclos de cultivo. Se observa un efecto en el tiempo, ya que hay una disminución en la presencia de ADN en la mayoría de los tratamientos. Al realizar el análisis estadístico, fue posible demostrar el efecto del tiempo para el tratamiento testigo $(\mathrm{p}=0,0162)$, así que la tendencia a la reducción de esporas para los tratamientos TSP y MM se pueden explicar, en parte, debido a ello; además, se puede relacionar con una pérdida de esporas vivas en las muestras debido a la germinación y su posterior degradación (Blum et al. 1997). En el tratamiento testigo, esta pérdida por germinación se puede adjudicar al menos a la presencia de la raíz de la planta hospedera, que provoca la germinación de las esporas, debido a la producción de sustancias dentro del contexto de los procesos co-evolutivos de estas 2 especies (Dixon 2009, Blouin 2017); por ello en el recipiente sin planta de mostaza (TSP) la pendiente (Tabla 4) es menor, sin embargo, es también sabido que otras especies no solo las crucíferas son capaces de inducir la germinación de las esporas, así como otras condiciones edáficas particulares (Dixon 2009).

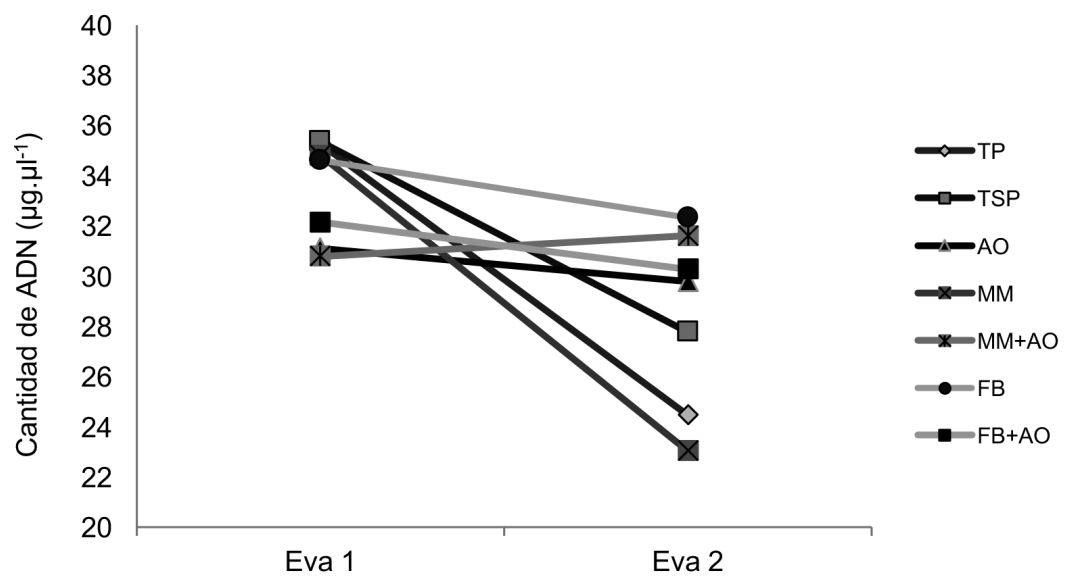

Figura 8. Comparación en los datos de rtPCR en suelo al inicio y al final de la etapa experimental.

Tabla 4. Ecuaciones y pendientes generadas entre las evaluaciones 1 y 2 para la variable rtPCR en el suelo por tratamiento.

\begin{tabular}{ll}
\hline Tratamiento & $\begin{array}{l}\text { Ecuación de } \\
\text { pendiente }\end{array}$ \\
\hline TP & $\mathrm{y}=-10,81 \mathrm{x}+46,09$ \\
TSP & $\mathrm{y}=-7,58 \mathrm{x}+42,94$ \\
$\mathrm{AO}$ & $\mathrm{y}=-1,33 \mathrm{x}+32,44$ \\
$\mathrm{MM}$ & $\mathrm{y}=-11,77 \mathrm{x}+46,58$ \\
$\mathrm{MM}+\mathrm{AO}$ & $\mathrm{y}=0,82 \mathrm{x}+29,96$ \\
FB & $\mathrm{y}=-2,29 \mathrm{x}+36,9$ \\
FB + AO & $\mathrm{y}=-1,87 \mathrm{x}+34,01$ \\
\hline
\end{tabular}

En el caso del tratamiento con MM, el valor de la pendiente es el mayor, de manera que se podría pensar en una mayor germinación y degradación de las esporas en presencia de MM, producto caracterizado por contar con una gran diversidad microbiana y, por tanto, producción de sustancias que podrían afectar la germinación de las esporas, así como su posterior degradación, logrando, de esta manera, la reducción de la cantidad esporas. Rolfe et al. (2016) indican que los flagelos de las esporas pueden ser dañados por una gran cantidad de factores, impidiendo de esta manera la infección de los pelos radicales. 
Por otro lado el tratamiento $\mathrm{MM}+\mathrm{AO}$, como se puede observar en la Tabla 4, fue el único en obtener una pendiente positiva, este dato es interesante, puesto que, al utilizar el abono orgánico junto con el MM, es probable que las esporas hayan germinado por el efecto del MM del abono orgánico de la planta hospedera o todas las posibles interacciones sistémicas, pero, además, contaron con los recursos y las condiciones necesarias que proporcionó el abono orgánico, para completar su ciclo de vida sobre las raíces vigorosas. En este sentido, Rolfe et al. (2016) indican que las agallas son una fuente metabólica que provee a Plasmodiophora brassicae con nutrientes tales como carbohidratos y aminoácidos. De ello, se deduce que la forma de aplicación del los bioles de MM es relevante y puede afectar el contenido de esporas de Plasmodiophora brassicae en el suelo, en el sentido de que, hacer las aplicaciones de MM directamente a la raíz de las plántulas por inmersión, puede ocasionar, como ya se dijo, lesiones en la raíz que la hagan más susceptible y junto con una mayor germinación de esporas $\mathrm{y}$ en presencia de un medio nutricionalmente adecuado, proporcionado por el abono orgánico, incrementar la cantidad de esporas en el suelo.

Un posible mejor uso del MM, en aras de disminuir la cantidad de esporas en el suelo, es aplicar el MM directamente al suelo antes de la siembra de la mostaza para así propiciar la germinación de las esporas sin la presencia del cultivo. En este sentido, Dixon (2009) indica que una vez germinadas las esporas, solo pueden recorrer muy cortas distancias en periodos de tiempo muy reducidos, por lo que se exponen a condiciones hostiles y adversas, antes de gastar sus reservas energéticas. Este periodo es entonces el más susceptible para Plasmodiophora brassicae y, por tanto, un factor importante a considerar al diseñar estrategias para el control, que tomen en cuenta el efecto benéfico de la aplicación de abono orgánico sobre el rendimiento del cultivo y los beneficios al suelo y el posible potencial del MM sobre la reducción de esporas en el suelo.

\section{LITERATURA CITADA}

Arteaga, M; Gárces, N; Guridi, F; Pino, J; López, A; Ménendez, J; Cartaya, O. 2006. Evaluación de las aplicaciones foliares de humus líquido en el cultivo del tomate var. Amalia en condiciones de producción. Cultivos Tropicales 27(3):95-101.

Auer, S; Ludwig-Müller, J. 2015. Biological control of clubroot (Plasmodiophora brassicae) by the endophytic fungus Acremonium alternatum. Journal of Endocytobiosis and Cell Research 26:43-49.

Bais, H; Weir, T; Perry, L; Gilroy, S; Vivanco, J. 2006. The Role of Root Exudates in Rhizosphere Interactions with Plants and Other Organisms. Annu Rev Plant Biol. 57:233-266.

Barrios, M; Buján, A; Debelis, S; Sokolowski, A; Blasón, A; Rodríguez, H; Gagey, M. 2014. Root Biomass/Total Ratio in Soybean (Glycine max) Under Two Tillage Systems. Terra Latinoamericana 32:221-230.

Bittner, M; Hilscherová, K; Gies, Y. 2007. Changes of AhR mediated activity of humic substances after irradiation. Environ. Intern. 3:812-816.

Björling, O. 2013. Plasmodiophora brassicae - host and environment interactions, Uppsala, Swedish, University of Agricultural Sciences. 33 p.

Blouin, M. 2017. Chemical communication: An evidence for co-evolution between plants and soil organisms. Applied Soil Ecology 123:409-415.

Blum, S; Lorenz, M; Wackernagel, W. 1997. Mechanism of Retarded DNA Degradation and Prokaryotic Origin of DNases in Nonsterile Soils. Appl. Microbiol. 20(4):513-521.

Bonanomi, G; Lorito, M; Vinale, F; Woo S. 2018 Organic Amendments, Beneficial Microbes, and Soil Microbiota: Toward a Unified Framework for Disease Suppression. Annual Review of Phytopathology 56:1-20.

Buysens, S; Heungens, K; Poppe, J; Hofte, M. 1996. Involvement of pyochelin and pyoverdin in suppression of Pythium-induced damping-off of tomato by Pseudomonas aeruginosa 7NSK2. Applied and Environmental Microbiology 62:865-871.

Cano, M. 2011. Interacción de microorganismos benéficos en plantas: Micorrizas, Trichoderma spp. y Pseudomonas spp. Actual Divulg Científica 14:15-31.

Castillo, J; Guerrero, O. 2008. Efecto de controladores biológicos sobre la hernia de las crucíferas en Tabio, Cundinamarca. Revista Inventum 5:30-40.

Castro, L; Murillo, M; Uribe, L; Mata, R. 2015. Inoculación al suelo con Pseudomonas fluorescens, Azospirillum oryzae, Bacillus subtillis y microorganismos de montaña (MM) y su efecto sobre un sistema de rotación soya-tomate bajo condiciones de invernadero. Agronomía Costarricense 39(3):21-36.

Deora, A; Gossen, BD; Amirsadeghi, S; McDonald, MR. 2015. A Multiplex qPCR Assay for Detection and 
Quantification of Plasmodiophora brassicae in Soil. Plant Disease 99(7):1002-1009.

Dixon, G. 2009. Plasmodiophora brassicae in its environment. Journal of Plant Growth Regulation 28(3):212-228.

Dixon, G. 2014. Clubroot (Plasmodiophora brassicae Woronin) an agricultural and biological challenge worldwide. Canadian Journal of Plant Pathology 36(1):5-18.

Durango, W. 2014. Efecto de dosis crecientes de enmiendas orgánicas en un andisol y un ultisol sobre la biomasa microbiana, respiración y actividad enzimática, en condiciones de invernadero. Tesis MSc. San José, Costa Rica, UCR. 137 p.

Einhorn, G; Bochow, H; Huber, J; Krebs, B. 1991. Methodological studies to detect anatagonists of the clubroot pathogen Plasmodiophora brassicae Wor. Phytopatholy Plant Protection 27(3):205-208.

Friberg, H; Lagerlöf, J; Hedlund, K; Rämert, B. 2008. Effect of earthworms and incorporation of grass on Plasmodiophora brassicae. Pedobiologia 52(1):29-39.

Garro, J. 2016. El suelo y los abonos orgánicos. Instituto Nacional de Innovación y Transferencia en Tecnología Agropecuaria (INTA). San José, Costa Rica. 106 p.

Imran, A; Siddiqui, S; Shaukat, S; Sheikh, H; Khan, A. 2006. Role of cyanide production by Pseudomonas fluorescens CHA0 in the suppression of root knot nematode, Mwloidogyne javanica in tomato. World J Microbiol Biotechnol. 22(6):641-650.

Kibblewhite, M; Ritz, K; Swift, M. 2008. Soil health in agricultural systems. Phil. Trans. R. Soc. B. 363:685-701.

Lockwood, J. 1988. Evolution of concepts associated with soilborne plant pathogens. Ann. Rev. Phytopathol. 26:93-121.

Lugtenberg, B; Kamilova, F. 2009. Plant-Growth-Promoting Rhizobacteria. Annu Rev. Microbiol. 63:541-556.

Moreno, A; García, G; Gutiérrez, P; Cano, R; Martínez, V; Márquez, M; Rodríguez, D. 2014. Desarrollo del cultivo de melón (Cucumis melo) con vermicompost bajo condiciones de invernadero. Ecosistemas y recursos 1(2):163-173.

Nandakumar, R; Babu, S; Viswanathan, R; Raguchander, T; Samiyappan, R. 2001. Induction of systemic resistance in rice against sheath blight disease by Pseudomonas fluorescens. Soil Biol Biochem. 33:603-612.

Neher, D. 2019. Compost And Plant Disease Suppression. BioCycle 60(8):22.

Neuhauser, C; Fargione, J. 2004. A mutualism-parasitism continuum model and its applicationto plantmycorrhizae interactions. Ecological Modelling 177:337-352.
Niwa, R; Kumei, T; Nomura, Y; Yoshida, S; Osaki, M; Ezawa, T. 2007. Increase in soil pH due to Ca-rich organic matter application causessuppression of the clubroot disease of crucifers. Soil Biology \& Biochemistry 39:778-785.

Orozco, R; Muñoz, R. 2012. Efecto de abonos orgánicos en las propiedades químicas del suelo y el rendimiento de la mora (Rubus adenotrichus) en dos zonas agroecológicas de Costa Rica. Tecnología en marcha 25(1):16-31.

Ramírez, C; Nienhuis, J. 2012. Cultivo protegido de hortalizas en Costa Rica. Tecnología En Marcha 25:10-20.

Reyes, J; Luna, R; Reyes, M; Suárez, G; Ulloa, C; Cabrera, D; Jhonn, G. 2016. Abonos orgánicos y su efecto en el crecimiento y desarrollo de la col (Brassica oleracea L.). Revista de Ciencias Biológicas y de La Salud, XVIII:28-32.

Reyes, I; Valery, A. 2015. Efecto de la fertilidad del suelo sobre la microbiota y la promoción del crecimiento del maíz (Zea mays 1.) con Azotobacter sppitle. Bioagro. 19(3):117-26.

Riascos, D; Ortiz E; Quintero, D; Montoya, L; Hoyos, L. 2011. Histopathological and morphological alterations caused by Plasmodiophora brassicae in Brassica oleracea L. Agronomía Colombiana 29(1):57-61.

Rolfe, S; Strelkov, S; Links, M; Clarke, W; Robinson, S; Djavaheri, M; Malinowski, R; Haddadi, P; Kagale, S; Parkin, I; Taheri, A; Borhan, M. 2016. The compact genome of the plant pathogen Plasmodiophora brassicae is adapted to intracellular interactions with host Brassica spp. BMC Genomics $17: 272$.

Ruiz, C; Russian, T; Tua, D. 2007. Efecto de la fertilización orgánica en el cultivo de la cebolla. Agronomía Tropical 57:7-14.

Sánchez-Bayo, F; Tennekes, H. 2017. Assessment of ecological risks of agrochemicals requires a new framework. Environ. Risk Assess. Remediat.1(3):20-28.

Schofield, E; Rowntree, J; Paterson, E; Brooker, R. 2018. Temporal Dynamism of Resource Capture: A Missing Factor in Ecology?. Trends in Ecology \& Evolution 33(4):277-286.

Strehlow, B; De Mol, F; Struck, C. 2015. Risk Potential of Clubroot Disease on Winter Oilseed Rape. Plant Disease 99(5):667-675.

Tanaka, S; Kochi, S; Kunita, H; Ito, S; Kameya-Iwaki, M. 1999. Biological mode of action of the fungicide, flusulfamide, against Plasmodiophora brassicae (clubroot). European Journal of Plant Pathology 105(6):577-584.

Valverde, F; Alvarado, S; Torres, C; Quishpe, J; Parra, R. 2011. Los abonos orgánicos en la productividad de papa (Solanum tuberosum L.). IV Congreso Ecuatoriano de la papa, Guaranda, Quito, Ecuador. 
Instituto Nacional Autónomo de Investigaciones Agropecuarias. $11 \mathrm{p}$.

Villalobos, E. 2001. Fisiología de la Producción de Los Cultivos Tropicales. Editorial Universidad de Costa Rica. San Pedro, San José Costa Rica. 227 p.

Wang, Y; Lou, W; Huang, Y; Xu, L; Yin, Y. 2017. Improved control of clubroot (Plasmodiophora brassicae) by a mixture of a fungicide and a plant defense inducer. Journal of Plant Diseases and Protection 124:67-71.

Willigen, P; Noordwijk, M. 1987. Root, plant production and nutrient use efficiency. Wageningen, Holanda. $282 \mathrm{p}$.
Yeoung, Y; Kim, J; Kim, B; Young, J; Yoon, C. 2003. Effects of beneficial antagonists Bacillus sp., Pseudomonas sp. and Trichoderma sp. on the control of clubroot of Chinese cabbage. Korean J Hort Sci Technol 21(3):194-198.

Zhang, W; Dick, W; Hoitink, H. 1996. Compost-Induced Systemic Acquired Resistance in Cucumber to Pythium Root Rot and Anthracnose. Phytopathology 86:1066-1070.

Todos los derechos reservados. Universidad de Costa Rica. Este artículo se encuentra licenciado con Creative Commons Reconocimiento-NoComercial-SinObraDerivada 3.0 Costa Rica. Para mayor información escribir a rac.cia@ucr.ac.cr 
